\title{
Conceptual Data Management and Communication for Smart Distribution System
}

\author{
J.J. Jamian ${ }^{\text {a, }}$ M.W. Mustafa ${ }^{\text {a, }}$ H. Mokhlis ${ }^{\text {b, }}$ M.A. Baharudin ${ }^{\text {a }}$ \\ ${ }^{a}$ Faculty of Electrical Engineering, Universiti Teknologi Malaysia. \\ ${ }^{\mathrm{b}}$ Faculty of Engineering, University of Malaya.
}

\begin{abstract}
Load adjustment is a new concept in smart grid system that can be used to balance the frequency of the system by adjusting their power consumption. However, the system required to have effective data communication management. The introduction of Smart Home System (which consists of Smart Appliance (SA) and Smart Meter (SM)), Smart Regional Server (SRS) and Smart Server (SS) as well as the messaging sequence between these entities will be used to manage data communication in smart distribution system. The communications between these devices will be via Power Line Communication (PLC) and WiMAX as a medium to transfer the data to the SRS. Transport Control Protocol (TCP) will increase the reliability of transferring data. By using the propose communication and data management technique, the use of load-balancing control to recover the frequency in a smart distribution grid system will be achieved.
\end{abstract}

Index Terms-- smart distribution gird; communication; data management; controllable load; messaging sequence.

\section{NOMENCLATURE}

\begin{tabular}{ll}
\hline \hline \multicolumn{1}{c}{ Term } & Abbreviation \\
\hline \hline External Energy Sources & EES \\
Electrical Vehicle & EV \\
Home Appliance & HA \\
Internet Protocol & IP \\
Network Interface Card & NIC \\
Power Line Communication & PLC \\
Smart Appliance & SA \\
Stream Control Transmission Protocol & SCTP \\
Smart Meter & SM \\
Smart Regional Server & SRS \\
Smart Server & SS \\
Transmission Control Protocol & TCP \\
\hline \hline
\end{tabular}

\section{INTRODUCTION}

The main component required by a power system to maintain a stable frequency is by balancing active power supply and demand. Without this balancing mechanism, the frequency of the system might collapse and cause system black-out. Commonly, load balancing is automatically conducted by the load frequency controller in a generator.
However, the balancing mechanism could be limited by some constraints such as stability of the generators, transmission line capacity limit and power losses [1-4]. If the generator does not able to fulfil the demand due to these constraints, load shedding might need to take place to avoid the system from collapsing [5-7]. Another technique of load frequency control is by controlling the total load consumption in a system. In this technique, the load is not connected or disconnected like load shedding method but instead, the load is controlled so that its power consumption can be adjusted whenever necessary in order to maintain the frequency stability. This technique is started to gain interest by researcher due to the introduction of controllable load and external energy sources such as Electric Vehicle (EV) and renewable energy (PV panel). In order to implement this technique, the use of advanced sensor, communication technologies and computing technique are necessity. However, beside the advancement of technologies, the utility or government also required to look on consumer educational aspect. If all consumers are willing to join in the power management scheme, it will give many benefits to the utility and consumer itself.

This paper presents the idea of how to make communication and data management successful between smart home system and the utility system. The main entities in the system are the Smart Server (SS), the Smart Regional Server (SRS), the Smart Meter (SM) and the Smart Appliances (SA). All these entities is communicating and transferring data between each other in order to make generation-load real power balancing achievable. The remaining of this paper is structured as follows. The following section introduces the detail of smart grid devices and the module which is proposed to enable two way communications. Section IV describes the process taken by smart devices in order to maintain the frequency of the system. Section V discusses the role of communication management to make this system successful and the paper ends by presenting a conclusion and future works.

\section{HYBRID COMMUNICATION MANAGEMENT}

The network topology of the system distribution is illustrated in Fig. 1. Since the communication and data transfer in this smart system involves the SS down to the SAs, these entities are required to have the capability to communicate and send data in both ways. The next subsection will explain in detail the proposed modules that can be implemented in appliances to make it possible for them to communicate. 


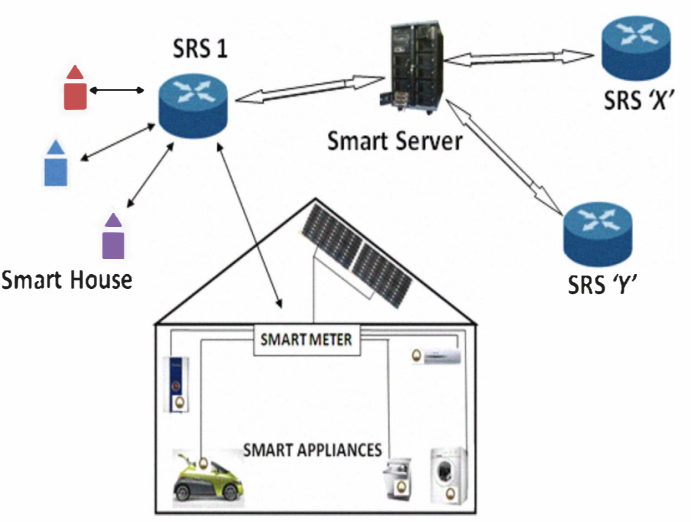

Fig. 1. Overall topology of the smart distribution system network.

\section{A. Module for Smart Appliances}

In order to allow the consumer to take part in power management, we need to have appliances which can communicate with the component at power supply side. In this paper, the smart appliance (SA) is define as appliances which are embedded with a module which would enable the home appliance (HA) itself to detect the state that it is in and schedules its task completion according to the decision by smart meter (SM). The structure of the proposed module is as depicted in Fig. 2 where it consists of the power metering unit, the sensing unit, the Network Interface Card and the controller unit. With this SA, we do not need to have a smart socket for the appliance to communicate with the SM as proposed in [8][9].

The power metering unit is the entity which calculates the power consumption of the appliance and sends the data to the controlling unit. The sensing unit is the entity that senses the current condition of the appliance. The Network Interface Card (NIC) is the entity which will modulate and send the data in the uplink direction and demodulate and transfer the information received in the downlink direction to the upper layers. The controller unit will act as the brain for this module. It will get the data from the power metering unit and send it to the SM via the NIC. It will also sense via the sensing unit and react accordingly. Furthermore, it receives commands from the SM via the NIC and executes the changes required by the SM. With the implementation of this module, the system will be fully automated. Plus, users can set their preferences in these modules so that when power reduction command is received, it will act according to the user preferences.

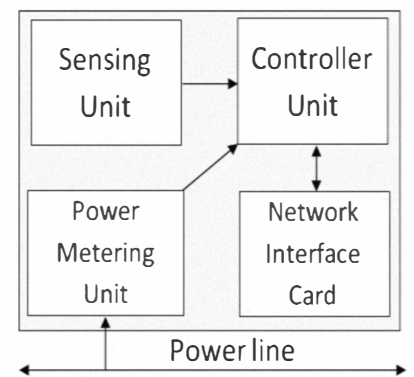

Fig. 2: Conceptual Module to be implemented into SAs.

\section{B. Types of Smart Home Appliances}

In general, the task for power metering unit and network interface card is same for all types of appliances while the others two units will be different depending on the types of load.

\section{1) Thermal Based Load}

For SAs with heat storage capability such as refrigerators and water heaters, it can be switched on or off for a certain period of time (seconds up to minutes) without affecting their acceptable temperature. In this case, the sensing unit will be used to sense the current temperature of the device and send it to the controller unit. The controller will analyze the data that has been forwarded by the sensing unit and make sure that the temperature is within the limit. If the temperature becomes lower than the acceptable value, the controller unit will turn on the appliance automatically.

\section{2) Operation Mode Based Load}

For the load that can function in multi mode operating conditions, the SM will ask the SA to change their mode when needed. However, the operation mode changes depend on the consumer settings. Air conditioner is one of the examples of SA unit under this category. So, the user can set the range of the operation for instance, from $19^{\circ} \mathrm{C}$ up to $25^{\circ} \mathrm{C}$ during day time, and the smart meter will nun the analysis to decide the suitable temperature that will be allowed. So, it will depend on consumer willingness and incentive from govemment. The sensing unit will sense the presence of a person in the area or the room. In example, the air conditioner will turn on when a person enters the room and turn off when the person leaves the room.

\section{3) Operating Time Based Load}

Washing machine and dishwasher are the examples of electrical devices that can delay or postpone their operation without giving a big impact to the consumer. So, the consumer only needs to set the completing time that is required by them and the SM will determine the suitable time of operation for the devices. For example, if the consumers want the dishwasher completing its job before he or she returns home at $5 \mathrm{pm}$, the SM will run the machine during light load. However, this type of load will be at lower priority of load changing if the remaining time for the device to complete the task is shorter. Table 1 shows the summary of the load categorization in the smart grid system.

\section{External Energy Sources (EES)}

The use of PV, EV and other energy devices in the home system will give tremendous benefit to the user. This external source is not only helping to stabilize the frequency of the system by supplying the power to the generation side, but also will generate some money from the power that has been supplied. For the deregulated power system, the electricity price is varied depending on the load condition. So, during the price for selling power (either from $\mathrm{PV}$ and $\mathrm{EV}$ ) is higher than buying power price, these energy resources can sell their power to the utility. On the other hand, if their price is lower than buying price, the PV can supply its power to its load (home) and get some support from the power system. If the 
house is totally disconnected from the utility due to some problems, the external resources still could supply the household with the collaboration with smart appliances. So, the SA will adjust their power consumption based on the availability of the energy in EES.

TABLE I. CHARACTERISTICS OF SMART LOAD

\begin{tabular}{|c|c|c|c|}
\hline Trpe of Load & Example & Criteria & Coumeat \\
\hline Thermal Bsed Load & $\begin{array}{l}\text { Refrigerators, witer } \\
\text { besters }\end{array}$ & $\begin{array}{l}\text { Tun "0a" or } \\
\text { "off" for a certain } \\
\text { period of timbe } \\
\text { nithoutd issuting } \\
\text { it main task. }\end{array}$ & $\begin{array}{l}\text { - needs to consider the } \\
\text { marg ginal time before the laad } \\
\text { can be turn on aggin after the } \\
\text { turn off process } \\
\text { - Frequency of switching that is } \\
\text { allowed }\end{array}$ \\
\hline $\begin{array}{l}\text { Operation Mode Based } \\
\text { Load }\end{array}$ & Air conditioner, fan & 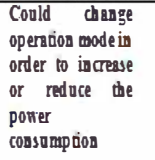 & $\begin{array}{l}\text { - Need to depend on consumer } \\
\text { willingness and incentive } \\
\text { from government. } \\
\text { - consumer eduction become } \\
\text { very important }\end{array}$ \\
\hline $\begin{array}{l}\text { Operating Time Based } \\
\text { Load }\end{array}$ & $\begin{array}{l}\text { Tisbing machine } \\
\text { and disb washer }\end{array}$ & 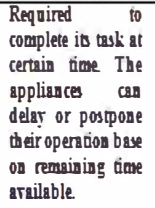 & $\begin{array}{l}\text { - Depending on time limit to } \\
\text { operate. } \\
\text { - Very useful fifthe remaining } \\
\text { time that is set ty user still } \\
\text { long. }\end{array}$ \\
\hline
\end{tabular}

\section{SMART POWER DISTRIBUTION SYSTEM OPERATION}

All smart devices in the system need to be managed properly in order to make the system reliable. With the collaboration between the SS, SRS, SA and EES, the frequency of the system can be maintained and the chances for the whole power system to blackout will be reduced.

\section{A. Frequency Drop Operation}

The Smart Server (SS) is used to sense the frequency changes that occur in the system by using the method proposed in [11]. After analysing and obtaining the amount of load to be reduced, the SS will refer to its database and determine the available amount of load that can be adjusted in each SRS and send the data signal to select SRSs. When the SRS receives the command to adjust the power consumption in its own region, it will forward the message to the selected SMs (houses). The selection process of SM is based on their availability of load reduction and maximum reduction on power losses.

After the SM gets the signal from SRS for adjusting their power consumption, it will ask the smart appliance to reduce their power consumption in order to improve the frequency of the system. Not only that, EES will also support the system by supplying generated power to the distribution system. So, a simple optimization technique like PSO is used to tackle this complex system. Once SA takes the action, the SA will update the latest condition to SRS through SM. The SS will also know the changes in SRS and update its own database.

\section{B. Frequency Increase Operation}

The same technique and communication management between SS, SRS and SM will be used for this condition. The only difference is the amount of power consumption needed to be adjusted. In previous cases, the load will be asked to reduce its power consumption either stopping the operation or changing its mode to the mode which uses low power. However, in this condition, the loads will be commanded to increase their power consumption like charging the EV and turning "on" the appliances which have delayed their task before this. The output from PV will not been supplied to the distribution system in this condition but it will be stored either by charging the EV or external batteries. Fig. 3 shows the summaries of operation for this system.

\section{COMMUNiCATION MANAGEMENT IN SMART SySTEM}

A network protocol for PLC which is called X10 is presented in [14]. This protocol only supports one way communication and does not have any error detection capabilities. Furthermore, the $60 \mathrm{~Hz}$ carrier frequency used in this protocol is affected by the interference from the $60 \mathrm{~Hz}$ signal as well as the attenuations from different load such as from computers and television [9]. An enhancement of this protocol was done in [9], however, it is still using one way communication. In order to achieve the smart grid requirements, a two way communication is needed [6][12]. Thus in this paper, a two-way network communication protocol is proposed.

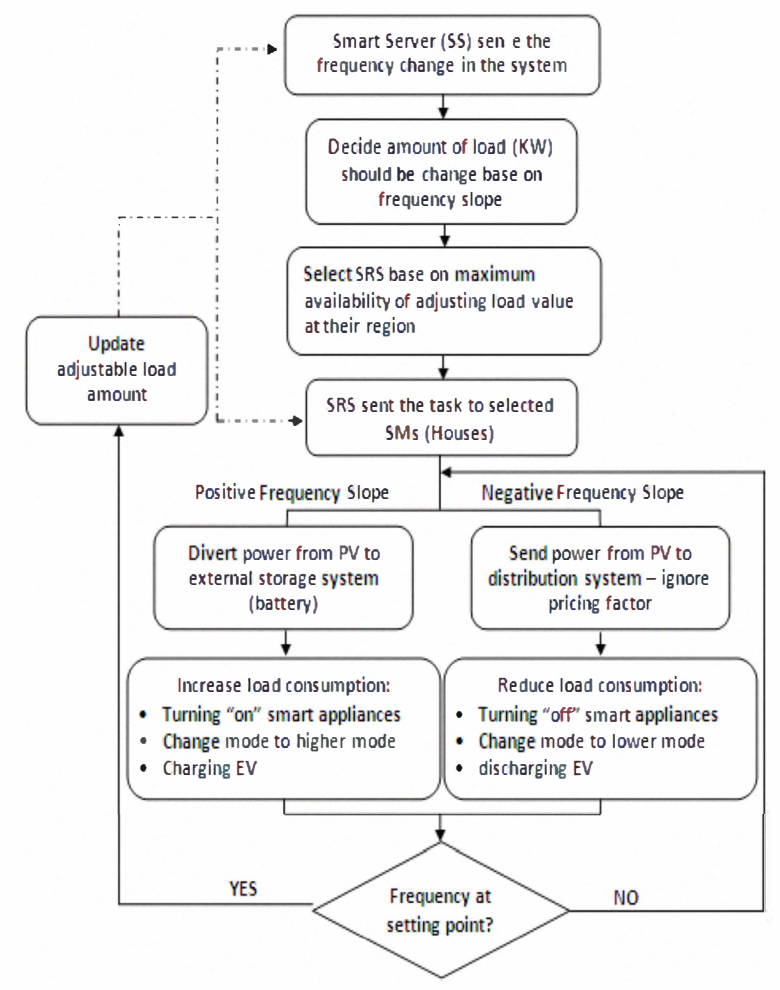

Fig. 3: Smart distribution system operation process

Although a simulation platform for smart metering system within one house which has its own network protocol which does two-way communication has been proposed in [10], but there is no mode control in their protocol. Considering such limitation, in this paper, a mode control scheme will be introduced and discussed in the next sub-section. 


\section{A. Protocol stacks of the entities in the system}

The data collection is one of the most crucial parts in this system. Data losses can disrupt the accuracy of the smart grid load distribution system. In [10], the authors introduced a three layer protocol stack which consists of an appliance layer at the topmost part, network layer in the middle and the physical layer at the bottom. According to [9], the network layer used in [10] is likely to be the X10 protocol [13]. An enhancement of this protocol is done in [9]. This stack is feasible enough for the communication within the household network. Conversely, this stack is not enough to prevent packet loss in a larger network. Due to the huge number of clients accessing and sending data to the designated server, congestion and collision which causes packet drops is bound to happen. The network layer, which mostly consists of the IP protocol, does not support packet retransmission and reordering. For this reason, we would like to propose the use of transport layer in the communication protocol stack. Transport layer protocols such as TCP and SCTP supports these capabilities, and can guarantee a reliable connection between the SMs and the SS.



Fig. 4: Protocol Stack of the System

The proposed protocol stack is as shown in Fig. 4. The transport layer, TCP in particular, will be added in the protocol stacks of the SM, SRS and SS. The network layer of the SAs can be either using a new network protocol which will be developed in future works.

\section{B. Message Exchange in Smart System}

In order to realize the proposed system, a new set of messaging is needed to replace the previous one way communication messages [9][14]. The authors in [10] has created their own two way messaging set, but mode setting was not included. Thus in this paper, mode setting messages will be introduced. There are several sets of messages depending on the entities involved in the conversation. These sets are: i)messages between SA and SM, ii) messages between SM and SRS and, iii) messages between SRS and SS. The packet defined in [10] was created on the network level. In this paper, two sets of packets structure will be porposed in order to cater for both in-home communication (SA to SM and vice versa) and for out-of-home communication (SM-SRS-SS communication). And for the in-home messaging, the packet will be created for the network layer whilst the out-of-home messaging will be created for the application layer since the TCP and IP protocol will be used at the transport and network layer.

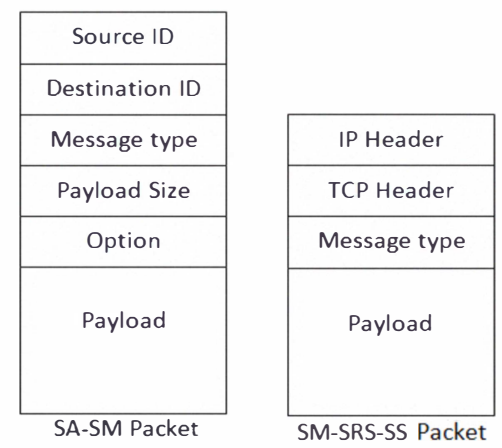

Fig. 5: Packet Structure

The proposed structure of the packets both SA-SM and SM-SRS-SS communication is as illustrated in Fig. 5. The message type for both packets will be the same. The messaging sequence in Fig. 6 will be triggered when the frequency of the grid changes as discussed in section 3 . The acceptable range of the frequency change is plus minus $0.1 \mathrm{~Hz}$. The SS will continually receive power consumption or generation data from the the lower entities when no frequency change happens. In this system, the power of command is shifted from up to down, from SS down towards SM. When the SS senses a frequency change, it will immediately calculate the amount of load to be adjusted and choose which SRS should be commanded to lower its load with the assumption that both SS and SRS has the same database. Then, when the SRS receives the request from SS, it will choose the target SMs according to its database so that the exact amount of load can be reduced. Then finally, the SRS will send requests to the designated SMs to command them to lower their load. When the load have been reduced, the SMs will send back a report update to SRS whom will also send back an update to the SS. The summary of this messaging sequence is as depicted in Fig. 6 and the types of messages is listed in Table 2 .

TABLE II. Packet Descriptions in Messaging Sequence

\begin{tabular}{|c|c|}
\hline Packet Type & Description \\
\hline XX_CONN REQ & $\begin{array}{l}\text { Requestto make connection from XX devices to WY devices } \\
\text { (XX/NY - SS, SRS, SM or SA) }\end{array}$ \\
\hline XX_CONN_ACK & Acknowledgementof "XX_CONN_REQ" \\
\hline SRS_L_CHANGE_REQ & Requestfor lood reduction from SRS to SM \\
\hline SM_L_CHANGE_REQ & Requestfor load reduction from SM to SA \\
\hline SM_TRANS_ID__REQ & $\begin{array}{l}\text { Requestfor transaction change from SM :O SA (in e. . To turn } \\
\text { the SA on or off, to change SA's mode) }\end{array}$ \\
\hline SA_CUR_STATE_REQ & $\begin{array}{l}\text { SA update currentstage : Total power consumption, Current } \\
\text { state appliance (on-off), State level available - power } \\
\text { adjustment that is still can be done, Timing available }\end{array}$ \\
\hline SM_TOT_AVAI_UPDATE & SM updates the total adjustable load in its juriscliction to SRS. \\
\hline SRS_TOT_AVAI_UPDATE & SRS updates the total adjustable load in its juriscdiction to SS. \\
\hline
\end{tabular}




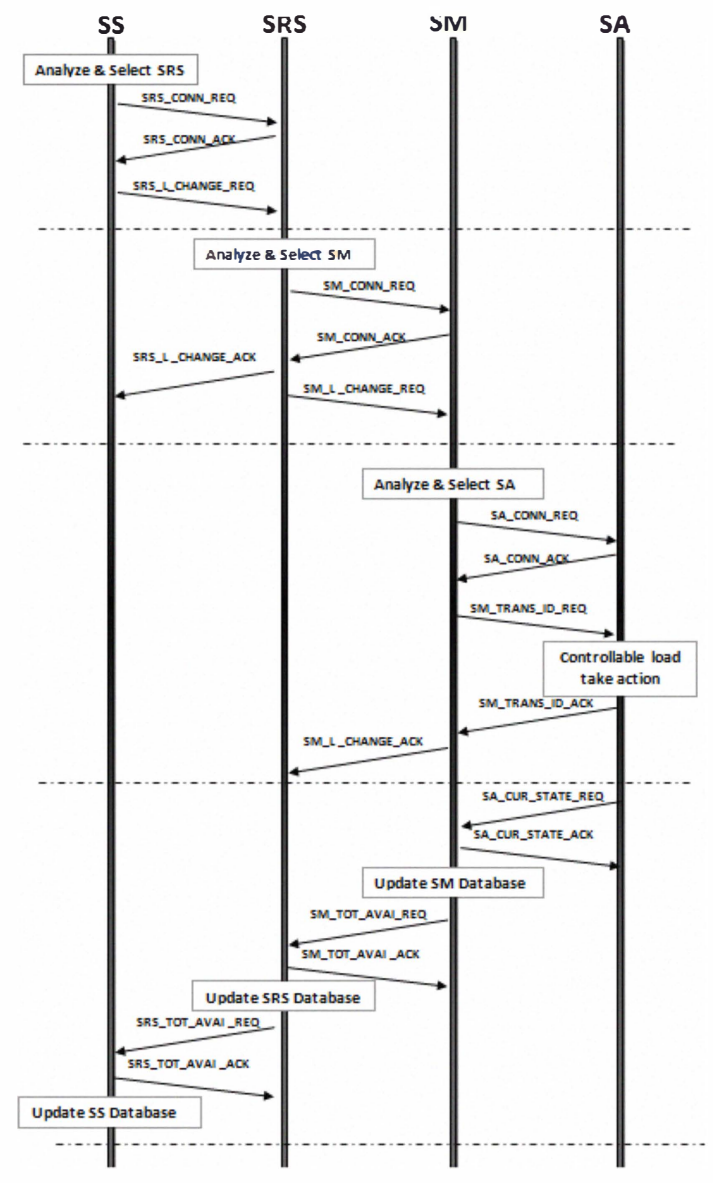

Fig. 6: Propose Messaging Sequence in Smart System

\section{CONCLUSION AND FUTURE WORKS}

In this paper, the smart grid system that consists of multiple smart devices has been introduced. All these devices have their own rule to balance the frequency. This condition creates the phenomena where the load side which is passive participant transform into an active participant in stabilizing the frequency changes in the grid. In summary, the following major contributions have been made in this paper.

1. The architecture for smart distribution grid system was proposed with the introduction of SRS. The main purpose of using the SRS in the system is to avoid any congestion that might occur if all smart meter need to directly send a signal to the SS. Furthermore, the SRS also makes the communication and data transfer simple.

2. The module introduced will enable two way communications and gives SM the ability to change the SA's mode. The use of Network Interface Card unit makes the SA capable to communicate with other devices.

3. The load for the system has been categorized based on their types of job. Some of the load can postpone their operation to help power system to improve the stability of frequency and some of them only can change their mode of operation. With this categorization, it is very helpful during decision making that needs to be done by the smart meter during load adjustment.
4. The use of TCP protocol in communication between SM, SRS and SS is to make the system more secure from data losses. The data losses can make the system "blur" and unable to participate in frequency adjustment. The messaging sequence gives an idea to future research on the packet data been transfer between all these smart devices in order to make the operation successful.

In conclusion, the controllable load will give many benefits if the communication and data transfer can be managed properly. Continuing researchers in this area includes the optimization technique that is use in the smart meter to make the selection of contribution for each load which will give significant impact to frequency stabilization and it challenges.

\section{REFERENCES}

[1] Verma, V.; Singh, B.; Chandra, A.; Al-Haddad, K.; , "Power Conditioner for Variable-Frequency Drives in Offshore Oil Fields," Industry Applications, IEEE Transactions on, vol.46, no.2, pp.731739, March-april 2010.

[2] Soder, Lennart; , "Explaining Power System Operation to Nonengineers," Power Engineering Review, IEEE , vol.22, no.4, pp.2527, April2002.s

[3] Fountas, N.A.; Hatziargyriou, N.D.; Orfanogiannis, C.; Tasoulis, A.; , "Interactive long-term simulation for power system restoration planning," Power Systems, IEEE Transactions on, vol.12, no.1, pp.6168, Feb 1997.

[4] Rajagopal, V.; Singh, B.; Kasal, G.K.; , "Electronic load controller with power quality improvement of isolated induction generator for small hydro power generation," Renewable Power Generation, IET , vol.5, no.2, pp.202-213, March 2011.

[5] Thalassinakis, E.J.; Dialynas, E.N.; Agoris, D.; , "Method Combining ANNs and Monte Carlo Simulation for the Selection of the Load Shedding Protection Strategies in Autonomous Power Systems," Power Systems, IEEE Transactions on , vol.21, no.4, pp.1574-1582, Nov. 2006.

[6] Seyedi, H.; Sanaye-Pasand, M.; , "New centralised adaptive loadshedding algorithms to mitigate power system blackouts," Generation, Transmission \& Distribution, IET , vol.3, no.1, pp.99-114, January 2009.

[7] Gungor, V.C.; Bin Lu; Hancke, G.P.; , "Opportunities and Challenges of Wireless Sensor Networks in Smart Grid," Industrial Electronics, IEEE Transactions on , vol.57, no.10, pp.3557-3564, Oct. 2010.

[8] Moslehi, K.; Kumar, R.; , "A Reliability Perspective of the Smart Grid," Smart Grid, IEEE Transactions on , vol.1, no.1, pp.57-64, June 2010.

[9] Rozeha A. Rashid; Mohd Adib Sarijari; Mohd Rozaini Abd Rahim; Tan Zun Yung;, "Flood Transmission based Protocol for Home Automation System via Power Line Communication," Proceedings of the International Conference on Computer and Communication Engineering, May 2008.

[10] Seunghyun Park; Hanjoo Kim; Hichan Moon; Jun Heo; Sungroh Yoon; , "Concurrent simulation platform for energy-aware smart metering systems," Consumer Electronics, IEEE Transactions on , vol.56, no.3, pp.1918-1926, Aug. 2010.

[11] Brooks, A.; Lu, E.; Reicher, D.; Spirakis, C.; Weihl, B.; , "Demand Dispatch," Power and Energy Magazine, IEEE , vol.8, no.3, pp.20-29, May-June 2010.

[12] Moslehi, K.; Kumar, R.; , "A Reliability Perspective of the Smart Grid," Smart Grid, IEEE Transactions on, vol.1, no.1, pp.57-64, June 2010.

[13] Zhang Yuejun; Wu Mingguang; , "Design of wireless remote module in $\mathrm{X}-10$ intelligent home," Industrial Technology, 2005. ICIT 2005. IEEE International Conference on, vol., no., pp.1349-1353, 14-17 Dec. 2005.

[14] Technical Note, "X-10 Communications Protocol and Power Line Interface", PSCO4 \& PSCO5. 